\title{
TYPE SEQUENCES OF ONE-DIMENSIONAL LOCAL ANALYTICALLY IRREDUCIBLE RINGS
}

\author{
VALENTINA BARUCCI and IOANA CRISTINA ŞERBAN
}

\begin{abstract}
We extend the notion of type sequence to rings that are not necessarily residually rational. Using this invariant we characterize different types of rings as almost Gorenstein rings and rings of maximal length.
\end{abstract}

\section{Introduction}

Let $(R, \mathfrak{m})$ be a one-dimensional local Cohen-Macaulay ring and let $\bar{R}$ be the integral closure of $R$ in its field of quotients. If we assume that $R$ is analytically irreducible, i.e., that $\bar{R}$ is a DVR (with a valuation $v$ ) and a finitely generated $R$-module, then the values of the elements of $R$ form a numerical semigroup $v(R)=\{v(a) \mid a \in R, a \neq 0\}=\left\{s_{0}=0, s_{1}, \ldots, s_{r-1}, s_{r}, \rightarrow\right\}$, where $s_{0}<s_{1}<\cdots<s_{r}$ and any integer $x, x \geq s_{r}$ is in $v(R)$ and the conductor $C=(R: \bar{R})$ is not zero.

If we further assume that $R$ is residually rational, i.e., that $k$, the residue field of $R$, is isomorphic to the residue field of $\bar{R}$, then $r=\ell_{R}(R / C)$ and a sequence of $r$ natural numbers $\left(t_{1}, \ldots, t_{r}\right)$ is naturally associated to $R, t_{i}=$ $\ell_{R}\left(\mathfrak{a}_{i}^{-1} / \mathfrak{a}_{i-1}^{-1}\right)$, where $\mathfrak{a}_{i}=\left\{x \in R \mid v(x) \geq s_{i}\right\}$. This sequence of natural numbers associated to the ring was for the first time considered by Matsuoka in [8]. As in [1] we call the sequence $\left(t_{1}, \ldots, t_{r}\right)$ the type sequence of $R$, t.s. $(R)$ for short. In particular the length $t_{1}=\ell_{R}\left(\mathrm{~m}^{-1} / R\right)$ is the Cohen Macaulay type of $R$ and it turns out that $\sum_{i=1}^{r} t_{i}=\ell_{R}(\bar{R} / R)$. A typical example of an analytically irreducible and residually rational ring is the ring of an algebraic curve singlularity with one branch.

It is well known that, for each one-dimensional local Cohen Macaulay ring with finite integral closure, the length $\ell_{R}(\bar{R} / R)$ is bounded below and above in the following way:

$$
\ell_{R}(R / C)+t-1 \leq \ell_{R}(\bar{R} / R) \leq \ell_{R}(R / C) t
$$

where again $C=(R: \bar{R})$ and $t$ is the CM type of $R$. 
The first inequality depends on the existence of a canonical ideal (cf. [2, Lemma 19 (e)]) and the second is proved in [3, Theorem 1]. If the ring $R$ is Gorenstein, i.e., of $\mathrm{CM}$ type 1 , then the two inequalities become equalities and $\ell_{R}(R / C)=\ell_{R}(\bar{R} / R)$. The rings which realize the minimal length for $\ell_{R}(\bar{R} / R)$, i.e., such that $\ell_{R}(R / C)+t-1=\ell_{R}(\bar{R} / R)$ have been introduced in [2] with the name of almost Gorenstein and recently revealed an interest in a geometric context (cf. [7]). On the other hand the rings which realize the maximal length for $\ell_{R}(\bar{R} / R)$ were characterized in [3] and also studied in [6].

In the analytically irreducible and residually rational case, there is a strict relation between the type sequence of $R$ and the length $\ell_{R}(\bar{R} / R)$. It is not surprising that the almost Gorenstein rings are characterized by a type sequence of the form $(t, 1,1, \ldots, 1)$ and those which realize the maximal length are characterized by a type sequence of the form $(t, t, \ldots, t)$, cf. [1] and [5, Theorem 1.7].

This paper deals with the analytically irreducible non-residually rational case. We have still a numerical semigroup $v(R)$ of values, but $k$, the residue field of $R$, is not isomorphic to $K$, the residue field of $\bar{R}$. The almost Gorenstein rings are characterized by a type sequence of the form $\left(t, n_{1}, \ldots, n_{r+l}\right)$ and the rings of maximal length by a type sequence of the form $\left(t, t n_{1}, \ldots, t n_{r+l}\right)$, where $n_{i}$ are the dimensions of certain $k$-vector subspaces of $K$ defined below.

As usual, if $\mathfrak{a}$ and $\mathfrak{b}$ are fractional ideals of $R$, then $\mathfrak{a}: \mathfrak{b}:=\{x \in Q(R) \mid$ $x \mathfrak{b} \subseteq \mathfrak{a}$, where $Q(R)$ is the field of quotients of $R, \mathfrak{a}^{-1}=R: \mathfrak{a}$ and $\mathfrak{a}$ is divisorial if $R:(R: \mathfrak{a}))=\mathfrak{a}$.

\section{The result}

In all this paper $R$ is a one-dimensional local analytically irreducible not necessarily residually rational ring. So the integral closure $\bar{R}$ is a DVR and $R$ has an associated semigroup of values:

$$
v(R)=\left\{s_{0}=0, s_{1}, \ldots, s_{r-1}, s_{r}=c, \rightarrow\right\},
$$

Denote by $X$ the generator of the maximal ideal of $\bar{R}$ and define the conductor of the ring as the natural number $N$ such that $R: \bar{R}=X^{N} \bar{R}$. Note that $N \geq c$, thus we can set $N=s_{r+l}=s_{r}+l=c+l$ for some $l \in \mathrm{N}$. In the residually rational case we have $N=c$. Thus in order to extend the definition of the type sequence to the non-residually rational case, some care is needed. As we shall see, in the general case the "right" definition will consist of a sequence of $r+l$ numbers.

Let us see the details. Consider the ideals of $R$ defined as

$$
\mathfrak{a}_{i}=\left\{x \in R \mid v(x) \geq s_{i}\right\}, \quad i \in\{0, \ldots, r+l\} .
$$


It is evident that $\mathfrak{a}_{0}=R, \mathfrak{a}_{1}=\mathfrak{m}$ and $\mathfrak{a}_{r+l}=R: \bar{R}$. Moreover, we have the following chain of inclusions:

$$
\mathfrak{a}_{r+l} \subset \cdots \subset \mathfrak{a}_{0}=\mathfrak{a}_{0}^{-1}=R \subseteq \mathfrak{a}_{1}^{-1} \subseteq \cdots \subseteq \mathfrak{a}_{r+l}^{-1},
$$

Note that whereas on the left side we have strict inclusions, on the right side, a priori, some of the inclusions could be equalities.

The following facts about the ideals $\mathfrak{a}_{i}$ are well known, but we recall them for the convenience of the reader:

Proposition 2.1. For every $i \in\{0, \ldots, r+l\}$, the ideals defined above have the following properties.

1. $\mathfrak{a}_{r+l}^{-1}=\bar{R}$;

2. $\mathfrak{a}_{i}$ is divisorial.

3. If $i>0$ then $\mathfrak{a}_{i}^{-1} \neq \mathfrak{a}_{i-1}^{-1}$ and hence $\ell_{R}\left(\mathfrak{a}_{i}^{-1} / \mathfrak{a}_{i-1}^{-1}\right) \geq 1$.

Proof. 1. As $\mathfrak{a}_{r+l}=X^{N} \bar{R}$, we have

$$
\begin{aligned}
\mathfrak{a}_{r+l}^{-1} & =R: X^{N} \bar{R}=X^{-N}(R: \bar{R}) \\
& =X^{-N} X^{N} \bar{R}=\bar{R} .
\end{aligned}
$$

2. As $\bar{R}=R: \mathfrak{a}_{r+l}^{-1}$, we have that $\bar{R}$ is divisorial as a fractional ideal of $R$. It follows that $X^{h} \bar{R}$ is divisorial for every $h \in \mathrm{N}$. This shows that $\mathfrak{a}_{i}$ is divisorial, since

$$
\mathfrak{a}_{i}=R \cap X^{s_{i}} \bar{R} .
$$

3. If $i>0$, then both $\mathfrak{a}_{i}$ and $\mathfrak{a}_{i-1}$ are divisorial. Thus, if $R: \mathfrak{a}_{i-1}=R: \mathfrak{a}_{i}$, then $\mathfrak{a}_{i-1}=R:\left(R: \mathfrak{a}_{i-1}\right)=R:\left(R: \mathfrak{a}_{i}\right)=\mathfrak{a}_{i}$, which is contradiciton.

Now we are ready to give our definition of type sequence of the ring $R$. For every $i \in\{1,2, \ldots, r+l\}$ let

$$
t_{i}(R):=\ell_{R}\left(\mathfrak{a}_{i}^{-1} / \mathfrak{a}_{i-1}^{-1}\right) .
$$

We call the sequence of numbers $\left(t_{1}(R), t_{2}(R), \ldots, t_{r+l}(R)\right)$ the type sequence of $R$, and we denote it by t.s. $(R)$.

As in the residually rational case we have that

$$
t_{1}(R)=\ell_{R}\left(\mathfrak{m}^{-1} / R\right)=: t(R)
$$

which is the Cohen-Macaulay type of $R$.

Note that for every $1 \leq i \leq r+l, \mathfrak{m} \mathfrak{a}_{i-1} \subseteq \mathfrak{a}_{i}$ and so $\mathfrak{a}_{i-1} / \mathfrak{a}_{i}$, is a $k$ vector space; let us denote it by $\left.V_{R}\left(s_{i-1}\right)\right)$. Since the inclusion $\mathfrak{a}_{i} \subset \mathfrak{a}_{i-1}$ is 
strict, $V_{R}\left(s_{i-1}\right) \neq 0$ and hence the number $n_{i-1}:=\operatorname{dim}_{k} V_{R}\left(s_{i-1}\right)$ is a positive integer. These vector spaces were considered also in [4] and can be defined not only for the ring $R$ but also for any fractional ideal of $R$. Let $F$ be such an ideal and $i \in \mathrm{N}$. Then

$$
F(i):=\{x \in F \mid v(x) \geq i\}
$$

is a fractional ideal of $R$ and we have $F(i) \subseteq F(j)$ for every $i \geq j$. The $R$-modules $F(i) / F(i+1)$ are also vector spaces over $k$, and we denote them by $V_{F}(i)$.

As we have outlined in the introduction, these vector spaces are very important for studying lengths for the analytically ireducible rings which are not residually rational. If $E \subseteq F$ are fractional ideals of $R$, then, in the residually rational case, $\ell_{R}(F / E)=\#\{v(F) \backslash v(E)\}$, cf. [8, Proposition 1]. In the nonresidually rational case we use the dimensions of the previous defined vector spaces as it was proved in [9].

Proposition 2.2 ([9, Proposition 11]). Let $E$ and $F$ be two fractional ideals of $R$ such that $E \subseteq F \subseteq \bar{R}$. Then there exists an $s \in \mathrm{N}$ such that

$$
\ell_{R}(F / E)=\sum_{r=0}^{s-1}\left[\operatorname{dim}_{k}\left(V_{F}(r)\right)-\operatorname{dim}_{k}\left(V_{E}(r)\right)\right] .
$$

We recall also another result which in this form appears in [9] and in fact it is an adapted version of [4, Proposition 3.5]. Observe that if $V$ and $W$ are two $k$-vector subspaces of $K$, where $k \subseteq K$ is a field extension, then $(V: W):=\{x \in K \mid x W \subseteq V\}$ is also a $k$-vector subspace of $K$.

Lemma 2.3 ([9, Lemma 3]). Let $k \subseteq K$ be an extension of fields with $n=\operatorname{dim}_{k} K<\infty$ and let $V \subset K$ be an $n-1$-dimensional $k$-vector subspace of $K$. Then for every $k$-vector subspace $W \subseteq K$ we have

$$
\operatorname{dim}_{k}(V: W)+\operatorname{dim}_{k}(W)=n .
$$

In order to prove our main theorem, we need the next result on the dimensions of previous defined vector spaces related to the fractional ideals $\mathfrak{a}_{i}^{-1}$, $i \in\{1, \ldots, r+l\}$.

Lemma 2.4. Let $n=\operatorname{dim}_{k} K$, where $k$ is the residue field of $R$ and $K$ is the residue field of $\bar{R}$. Then:

1. $\operatorname{dim}_{k}\left(V_{\mathfrak{a}_{i}^{-1}}\left(N-1-s_{i-1}\right)\right)=n$;

2. $\operatorname{dim}_{k}\left(V_{\mathfrak{a}_{i-1}^{-1}}\left(N-1-s_{i-1}\right)\right) \leq n-n_{i-1}$; 
Proof. Let us prove the first assertion. Fix an $i \in\{1, \ldots, r+l\}$. Then for any $\gamma \in K$ we have that

$$
\gamma X^{N-1-s_{i-1}} \mathfrak{a}_{i} \subseteq X^{N-1+\left(s_{i}-s_{i-1}\right)} \bar{R} \subseteq X^{N} \bar{R} \subseteq R .
$$

Therefore we have

$$
\gamma X^{N-1-s_{i-1}} \in R: \mathfrak{a}_{i}=\mathfrak{a}_{i}^{-1}
$$

for every $\gamma \in K$. Thus $K X^{N-1-s_{i-1}} \subseteq R: \mathfrak{a}_{i}$. It follows that $V_{\mathfrak{a}_{i}^{-1}}(N-1-$ $\left.s_{i-1}\right) \simeq K$ and this is of dimension $n$ over $k$.

Now we can prove the second assertion. It is easy to see that

$$
\gamma X^{N-1-s_{i-1}} \mathfrak{a}_{i-1} \subseteq R \Longleftrightarrow \gamma V_{R}\left(s_{i-1}\right) \subseteq V_{R}(N-1)
$$

which is of course further equivalent to $\gamma \in V_{R}(N-1): V_{R}\left(s_{i-1}\right)$.

Thus we have that:

$$
\gamma \in V_{a_{i-1}^{-1}}\left(N-1-s_{i-1}\right) \Longleftrightarrow \gamma \in\left(V_{R}(N-1): V_{R}\left(s_{i-1}\right)\right)
$$

Then we can conclude that:

$$
\operatorname{dim}_{k}\left(V_{a_{i-1}^{-1}}\left(N-1-s_{i-1}\right)\right)=\operatorname{dim}_{k}\left(V_{R}(N-1): V_{R}\left(s_{i-1}\right)\right) .
$$

As $V_{R}(N-1)$ is a proper subspace of $K$, we can find a $k$-vector subspace $U \subset K$ of codimension 1 such that $V_{R}(N-1) \subseteq U$ and so

$$
\begin{aligned}
\operatorname{dim}_{k}\left(V_{R}(N-1): V_{R}\left(s_{i-1}\right)\right) & \leq \operatorname{dim}_{k}\left(U: V_{R}\left(s_{i-1}\right)\right) \\
& =n-\operatorname{dim}_{k}\left(V_{R}\left(s_{i-1}\right)\right) \\
& =n-n_{i-1},
\end{aligned}
$$

where for the first equality we have used Lemma 2.3.

We shall see now certain upper and lower bounds for $t_{i}(R)$, which generalize [8, Proposition 3].

Proposition 2.5. For every $i \in\{1, \ldots, r+l\}$ :

$$
n_{i-1} \leq t_{i}(R) \leq t(R) n_{i-1} .
$$

Proof. To show the upper bound we shall use [3, Lemma 1]. This affirms that, if we have two ideals of the ring $R, I_{1}$ and $I_{2}$ such that $I_{1} \subseteq I_{2}$ and $I_{2} / I_{1}$ is a simple $R$-module, then

$$
\ell_{R}\left(R: I_{1} / R: I_{2}\right) \leq t(R) .
$$


We are using this result for our ideals $\mathfrak{a}_{i} \subseteq \mathfrak{a}_{i-1}$. The $R$-module $\mathfrak{a}_{i-1} / \mathfrak{a}_{i}$ is not simple, but it is in fact a $k$-vector space of finite dimension equal to $n_{i-1}$. Then we can apply [3, Lemma 1] $n_{i-1}$ times and we conclude the proof for the upper bound.

Now we want to show the lower bound. Using Proposition 2.2 we have that

$$
\ell_{R}\left(\mathfrak{a}_{i}^{-1} / \mathfrak{a}_{i-1}^{-1}\right) \geq \operatorname{dim}_{k}\left(V_{\mathfrak{a}_{i}^{-1}}\left(N-1-s_{i-1}\right)\right)-\operatorname{dim}_{k}\left(V_{\mathfrak{a}_{i-1}^{-1}}\left(N-1-s_{i-1}\right)\right)
$$

By Lemma 2.4, we get:

$$
t_{i}(R):=\ell_{R}\left(\mathfrak{a}_{i}^{-1} / \mathfrak{a}_{i-1}^{-1}\right) \geq n-\left(n-n_{i-1}\right)=n_{i-1} .
$$

Similarly to the residually rational case, we can characterize rings of minimal and maximal length by their type sequences.

THEOREM 2.6. Let $n_{i}=\operatorname{dim}_{k}\left(\mathfrak{a}_{i} / \mathfrak{a}_{i-1}\right)$. Then

1. $R$ is almost Gorenstein if and only if

$$
\text { t.s. }(R)=\left(t(R), n_{1}, n_{2}, \ldots, n_{r+l-1}\right) \text {. }
$$

2. $R$ is of maximal length if and only if

$$
\text { t.s. }(R)=\left(t(R), t(R) n_{1}, t(R) n_{2}, \ldots, t(R) n_{r+l-1}\right) .
$$

Proof. Recall that the almost Gorenstein property means (cf. [2, Definition-Proposition 20]) that

$$
\ell_{R}(\bar{R} / R)=\ell_{R}\left(R / \mathfrak{a}_{r+l}\right)+t(R)-1 .
$$

Equation (23) is equivalent to:

$$
\sum_{i=1}^{r+l} t_{i}(R)=\sum_{i=0}^{r+l-1} n_{i}+t(R)-1
$$

As $n_{0}=\operatorname{dim}_{k}\left(V_{R}\left(s_{0}\right)\right)=\operatorname{dim}_{k}(R / \mathfrak{m})=1$ and $t_{1}(R)=t(R)$, the previous equation is equivalent to:

$$
\sum_{i=2}^{r+l} t_{i}(R)=\sum_{i=1}^{r+l-1} n_{i}
$$

Thus, the conclusion follows using Proposition 2.5 which claims that $n_{i-1} \leq t_{i}$, so the previous equality holds if and only if $t_{i}=n_{i-1}$ for every $i, 2 \leq i \leq r+l$. 
The ring $R$ is of maximal length if and only if

$$
\ell_{R}(\bar{R} / R)=t(R) \ell_{R}\left(R / \mathfrak{a}_{r+l}\right) .
$$

And this is further equivalent to

$$
\sum_{i=1}^{r+l} t_{i}(R)=t(R) \sum_{i=0}^{r+l-1} n_{i}=\sum_{i=0}^{r+l-1} t(R) n_{i} .
$$

By Proposition 2.5, $t_{i} \leq t(R) n_{i-1}$ for every $i \in\{1, \ldots, r+l-1\}$, and we know also that $n_{0}=1$, so the previous equality holds if and only if $t_{i}=t(R) n_{i-1}$ for every $i \in\{1, \ldots, r+l\}$.

As a consequence of Theorem 2.6, 1, and of the fact that a Gorenstein (Kunz) ring is an almost Gorenstein ring of type 1 (2, respectively), we can draw the following conclusion.

COROllary 2.7.

1. $R$ is Gorenstein if and only if t.s. $(R)=\left(1, n_{1}, \ldots, n_{r+l-1}\right)$,

2. $R$ is Kunz if and only if t.s. $(R)=\left(2, n_{1}, \ldots, n_{r+l-1}\right)$.

Note that the proof for Gorensteiness could also have been obtained in a direct manner. Indeed, if $R$ is Gorenstein, then the ring itself is a canonical ideal, and so for every $i \in\{1, \ldots, r+l\}$,

$$
\begin{aligned}
t_{i}(R) & =\ell_{R}\left(\left(R: \mathfrak{a}_{i}\right) / R: \mathfrak{a}_{i-1}\right) \\
& =\ell_{R}\left(\left(R:\left(R: \mathfrak{a}_{i-1}\right)\right) /\left(R:\left(R: \mathfrak{a}_{i}\right)\right)\right)=\ell_{R}\left(\mathfrak{a}_{i-1} / \mathfrak{a}_{\mathfrak{i}}\right) \\
& =\operatorname{dim}_{k} V_{R}\left(s_{i-1}\right)=n_{i-1} .
\end{aligned}
$$

\section{Examples}

Example 1. Consider the following subring of the ring of power series $\mathrm{Q}(\sqrt{2}, \sqrt{3})[[X]]$.

$$
R=\mathrm{Q}+X^{3} \mathrm{Q}(\sqrt{2}, \sqrt{3})+X^{4} \mathrm{Q}(\sqrt{2}, \sqrt{3})+X^{5} \mathrm{Q}(\sqrt{2})+X^{6} \mathrm{Q}(\sqrt{2}, \sqrt{3})[[X]]
$$

In this example, $k=\mathrm{Q}$ and $K=\mathrm{Q}(\sqrt{2}, \sqrt{3})$. According to the notation of previous section, we have $n_{0}=1, n_{1}=4, n_{2}=4, n_{3}=2$. Moreover, since

$$
\begin{aligned}
& \mathfrak{a}_{1}^{-1}=\mathrm{Q}(\sqrt{2})+X^{3} \mathrm{Q}(\sqrt{2}, \sqrt{3})[[X]], \\
& \mathfrak{a}_{2}^{-1}=\mathrm{Q}(\sqrt{2})+X^{2} \mathrm{Q}(\sqrt{2}, \sqrt{3})[[X]], \\
& \mathfrak{a}_{3}^{-1}=\mathrm{Q}(\sqrt{2})+X \mathrm{Q}(\sqrt{2}, \sqrt{3})[[X]], \\
& \mathfrak{a}_{4}^{-1}=\mathrm{Q}(\sqrt{2}, \sqrt{3})[[X]],
\end{aligned}
$$


we get $t=t_{1}=3, t_{2}=4, t_{3}=4, t_{4}=2$, so that the type sequence is $\left(t, n_{1}, n_{2}, n_{3}\right)$ and the ring is almost Gorenstein.

EXAMPLE 2. A ring of maximal length.

$$
R=\mathrm{R}+X^{3} i \mathrm{R}+X^{6} \mathrm{R}+X^{9} \mathrm{C}[[X]]
$$

Here $k=\mathrm{R}, K=\mathrm{C}, n_{0}=n_{1}=n_{2}=1$ and since

$$
\begin{aligned}
& \mathrm{a}_{1}^{-1}=\mathrm{R}+X^{3} i \mathrm{R}+X^{6} \mathrm{C}[[X]], \\
& a_{2}^{-1}=\mathrm{R}+X^{3} \mathrm{C}[[X]], \\
& \mathrm{a}_{3}^{-1}=\mathrm{C}[[X]],
\end{aligned}
$$

we get $t=t_{1}=5, t_{2}=5, t_{3}=5$, so that the type sequence is $\left(t, t n_{1}, t n_{2}\right)$ and the ring is of maximal length.

The examples above are generalized semigroup rings, GSR for short, i.e., rings of the form

$$
k+X V_{1}+\cdots+X^{N-1} V_{N-1}+X^{N} K[[X]]
$$

where $V_{i}$ are $k$-vector subspaces of $K$. To every one-dimensional analytically irreducible ring $R$ can be associated a GSR $\widetilde{R}$, as in [9]. More precisely $\widetilde{R}$ is the subring of $K[[X]]$ defined, with the notation of the previous section, as

$$
\widetilde{R}:=\sum_{i \geq 0} V_{R}(i) X^{i}
$$

That is in fact the generalization of the way of associating to $R$, in the residually rational case, the semigroup ring $k[[S]]$, where $S=v(R)$. Observe however that the type sequence of $R$ and its associated GSR is not always the same. For example, if $R=k\left[\left[X^{4}, X^{6}+X^{7}, X^{10}\right]\right]$, with characteristic of $k$ unequal to 2 , then the associated GSR is $k\left[\left[X^{4}, X^{6}, X^{11}, X^{13}\right]\right]$, which has type sequence $(3,1,1,1)$. On the other hand the type sequence of $R$ is $(2,2,1,1)$, in fact $a_{1}^{-1}$ contains no element with value 2 , but $a_{2}^{-1}$ contains $X^{2}-X^{3}$. However we can prove that:

PROPOSITION 3.1. The ring $R$ is almost Gorenstein if and only if the associated GSR $\widetilde{R}$ is almost Gorenstein and type $(R)=\operatorname{type}(\widetilde{R})$.

Proof. Let $\omega$ be a canonical ideal of $R, R \subseteq \omega \subseteq \bar{R}$ (cf. [2] for the definition and the existence). Then, by [9, Theorem 17], $\widetilde{\omega}=\sum_{i \geq 0} V_{\omega}(i) X^{i}$ is a canonical ideal of $\widetilde{R}$. Moreover, by [9, equation (24) and Lemma 16], we have:

$$
\ell_{R}(\omega / R)=\ell_{\widetilde{R}}(\widetilde{\omega} / \widetilde{R}) \geq \operatorname{type}(\widetilde{R})-1 \geq \operatorname{type}(R)-1
$$


By [2, Definition-Proposition 20] we get that $R$ is almost Gorenstein if and only if $\ell_{R}(\omega / R) \geq \operatorname{type}(R)-1$. Thus $R$ is almost Gorenstein if and only if both inequalities of (29) are equalities, that is if and only if $\widetilde{R}$ is almost Gorenstein and type $(R)=\operatorname{type}(\widetilde{R})$.

We shall give now an example of computing the type sequence of a ring which is not a GSR and the length $\ell_{R}(\bar{R} / R)$ is not minimal neither maximal.

Example 3. Let $R=\mathrm{R}\left[\left[i X^{3}+X^{4}, X^{5}, i X^{10}+X^{11}, X^{16}\right]\right]$. For this ring $k=\mathrm{R}$ and $K=\mathrm{C}$. Observe that $R$ is not a GSR, but we can compute its associated GSR. First let us try to compute the type sequence of $R$. After some computations we can write $R$ as:

$$
\begin{aligned}
R=\mathrm{R} & +\left(i X^{3}+X^{4}\right) \mathrm{R}+X^{5} \mathrm{R}+\left(-X^{6}+2 i X^{7}+X^{8}\right) \mathrm{R} \\
& +\left(i X^{8}+X^{9}\right) \mathrm{R}+\left(-i X^{9}+3 i X^{11}+X^{12}\right) \mathrm{R}+X^{10} \mathrm{R} \\
& +\left(i X^{10}+X^{11}\right) \mathrm{R}+\left(-X^{11}+2 i X^{12}\right) \mathrm{R}+\left(X^{12}-2 X^{14}\right) \mathrm{R} \\
& +X^{13} \mathrm{R}+\left(i X^{13}+X^{14}\right) \mathrm{R}+i X^{14} \mathrm{R}+X^{15} \mathrm{C}[[X]] .
\end{aligned}
$$

Thus for the ring $R$ we have: the conductor of the ring $N=15$ and $n_{0}=1$, $n_{1}=1, n_{2}=1, n_{3}=1, n_{4}=1, n_{5}=1, n_{6}=2, n_{7}=1, n_{8}=1$, $n_{9}=2, n_{10}=1$. We compute now the inverses of the ideals which appear in the definition of the type sequence.

$$
\begin{aligned}
& \mathrm{m}^{-1}=\mathrm{R}+\left(i X^{3}+X^{4}\right) \mathrm{R}+X^{5} \mathrm{R}+\left(-X^{6}+2 i X^{7}+X^{8}\right) \mathrm{R} \\
&+\left(X^{7}-2 X^{9}\right) \mathrm{R}+\left(i X^{8}+X^{9}\right) \mathrm{R}+\left(-i X^{9}+3 i X^{11}+X^{12}\right) \mathrm{R} \\
&+X^{10} \mathrm{R}+\left(i X^{10}+X^{11}\right) \mathrm{R}+X^{11} \mathrm{R}+X^{12} \mathrm{C}[[X]] . \\
& \mathfrak{a}_{2}^{-1}=\mathrm{R}+\left(i X^{3}+X^{4}\right) \mathrm{R}+X^{5} \mathrm{R}+\left(-X^{6}+2 i X^{7}+X^{8}\right) \mathrm{R} \\
&+\left(X^{7}-2 X^{9}\right) \mathrm{R}+\left(i X^{8}+X^{9}\right) \mathrm{R}+X^{9} i \mathrm{R}+X^{10} \mathrm{C}[[X]] . \\
& \mathfrak{a}_{3}^{-1}=\mathrm{R}+\left(i X^{3}+X^{4}\right) \mathrm{R}+X^{5} \mathrm{R}+\left(-X^{6}+2 i X^{7}+X^{8}\right) \mathrm{R}+X^{7} \mathrm{R} \\
& \\
&+\left(i X^{7}+X^{8}\right) \mathrm{R}+X^{8} i \mathrm{R}+X^{9} \mathrm{C}[[X]] . \\
& \mathfrak{a}_{4}^{-1}=\mathrm{R}+\left(i X^{3}+X^{4}\right) \mathrm{R}+X^{5} \mathrm{R}+X^{6} \mathrm{R}+X^{7} \mathrm{C}[[X]] . \\
& \mathfrak{a}_{5}^{-1}=\mathrm{R}+\left(i X^{3}+X^{4}\right) \mathrm{R}+X^{5} \mathrm{R}+X^{6} \mathrm{C}[[X]] . \\
& \mathfrak{a}_{6}^{-1}=\mathrm{R}+\left(i X^{3}+X^{4}\right) \mathrm{R}+X^{5} \mathrm{C}[[X]] . \\
& \mathfrak{a}_{7}^{-1}=\mathrm{R}+\left(i X^{2}-X^{3}\right) \mathrm{R}+X^{3} i \mathrm{R}+X^{4} \mathrm{C}[[X]] \\
& \mathfrak{a}_{8}^{-1}=\mathrm{R}+X^{2} i \mathrm{R}+X^{3} \mathrm{C}[[X]] .
\end{aligned}
$$




$$
\begin{aligned}
& \mathrm{a}_{9}^{-1}=\mathrm{R}+X^{2} \mathrm{C}[[X]] . \\
& \mathfrak{a}_{10}^{-1}=\mathrm{R}+X \mathrm{C}[[X]] . \\
& \mathrm{a}_{11}^{-1}=\mathrm{C}[[X]]=\bar{R} .
\end{aligned}
$$

Then: $t_{1}=\ell_{R}\left(\mathrm{~m}^{-1} / R\right)=3, t_{2}=1, t_{3}=2, t_{4}=1, t_{5}=1, t_{6}=1, t_{7}=3$, $t_{8}=1, t_{9}=1, t_{10}=2, t_{11}=1$.

The associated GSR of $R$ is $\widetilde{R}=\mathrm{R}\left[\left[i X^{3}, X^{5}, i X^{10}, i X^{17}\right]\right]$. After computations we have that the type sequence of $\widetilde{R}$ is $\left(t_{1}=3, t_{2}=1, t_{3}=2, t_{4}=\right.$ $\left.1, t_{5}=1, t_{6}=1, t_{7}=3, t_{8}=1, t_{9}=1, t_{10}=2, t_{11}=1\right)$. We observe that in this case the type sequence of $R$ is equal to the type sequence of its associated GSR.

\section{REFERENCES}

1. Barucci, V., Dobbs, D. E., and Fontana M., Maximality properties in numerical semigroups and applications to one-dimensional analytically irreducible local domains, Mem. Amer. Math. Soc. 125 (1997).

2. Barucci, V., and Fröberg, R., One-dimensional almost Gorenstein rings, J. Algebra 188 (1997), $418-442$.

3. Brown, W. C., and Herzog, J., One dimensional local rings of maximal and almost maximal length, J. Algebra 151 (1992), 332-347.

4. Campillo, A., Delgado, F., and Kiyek, K., Gorenstein property and symmetry for onedimensional local Cohen-Macaulay rings, Manuscripta Math. 83 (1994), 405-423.

5. D'Anna, M., Canonical module and one-dimensional analytically irrreducible Arf domains, pp. 215-225 in: Commutative Ring Theory (Proc. Fés 1995), Lecture Notes in Pure and Appl. Math. 185, Dekker, New York 1997.

6. Delfino, D., On the inequality $\lambda(\bar{R} / R) \leq t(R) \lambda(R / \mathfrak{S})$ for one-dimensional local rings, J. Algebra 169 (1997), 332-342.

7. Kleiman, S., Martins, R. V., The canonical model of a singular curve, Geom. Dedicata 139 (2009), 139-166.

8. Matsuoka, T., On the degree of singularity of one-dimensional analytically irreducible Noetherian local rings, J. Math. Kyoto Univ. 11 (1971), 485-494.

9. Şerban, I. C., On canonical ideals of one-dimensional local analytically irreducible rings, Comm. Algebra 36 (2008), 4263-4275.

DIPARTIMENTO DI MATEMATICA

UNIVERSITÀ DI ROMA "LA SAPIENZA"

P.LE ALDO MORO 2

00185 ROMA

ITALY

E-mail: barucci@mat.uniroma1.it ioanase@yahoo.com 